\title{
Effects of Marigold Cultivation on Microbial Diversity of Tobacco-planting Soil
}

\author{
Song Jin ${ }^{1}$, a Xi Gao², Zebin Chen ${ }^{1}$, Yuan Su ${ }^{3}$, Jiani Liu ${ }^{1}$, Lei Yu ${ }^{4}$, Dingkang Wang', \\ Shengguang $\mathrm{Xu}^{5, \mathrm{~b}}, *$ \\ ${ }^{1}$ College of Agronomy, Kunming University, Kunming, Yunnan Province, China. \\ ${ }^{2}$ College of Plant Protection, Yunnan Agricultural University, Kunming, Yunnan Province, China. \\ ${ }^{3}$ Key Laboratory of Characteristic Biological Resource Development and Utilization of Colleges and \\ Universities in Yunnan Province, Kunming, Yunnan Province, China. \\ ${ }^{4}$ Yunnan Urban Characteristic Agriculture Engineering Technology Research Center, Kunming, Yunnan \\ Province, China. \\ ${ }^{5}$ Yunnan Institute of Biological Carbon Engineering Research Center, Kunming, Yunnan Province, China. \\ aibmjs@163.com, bsgxu2011@126.com \\ *Corresponding author
}

Keywords: high-throughput sequencing; marigold cultivation; tobacco-planting soil; microorganism; diversity

\begin{abstract}
With Yunyan 87 as the research material, this paper used Illumina MiSeq high throughput sequencing technology to study the effect of marigold cultivation on microbial diversity of tobacco planting soil under two kinds of planting patterns. The results showed that the diversity of bacterial Alpha was represented as bacterial abundance CC3.2 > AB3.2 > BB3.2 and bacterial diversity BB3.2 > AB3.2 > CC3.2. The diversity of fungal Alpha was represented as fungi abundance BB3.2 > AB3.2 > CC3.2 and fungi diversity BB3.2>AB3.2>CC3.2. At the level of genus, the dominant bacteria of three samples were similar; three kinds of bacteria with higher abundances were Kaistorbacte, Marinobacter and Nitrososphaera. There were only small differences in the abundances of the same genus. The content of Nitrososphaera in CC3.2 was lower than that in AB3.2 and BB3.2 by $0.80 \%$ to $0.91 \%$. The three samples had similar dominant fungi, but the abundances of fungi varied greatly. After the planting of marigold, the abundance of Aspergillus increased from the original number of $0.89 \%$ to values between $5.61 \%$ and $7.70 \%$. Compared with single cropping of tobacco, marigold inter planting with tobacco increased the abundance by 8.6 times. The abundance of Sordariales sp rose from the original number of 3.75\% to values between $4.09 \%$ and $5.03 \%$ after marigold cultivation. The abundance of Corynascella was between $6.24 \%$ and $7.39 \%$ under monoculture, and sharply decreased to $0.04 \%$ under the condition of tobacco inter planting with marigold. The decrease rate was over $99 \%$.
\end{abstract}

\section{Introduction}

As one of the most important commercial crops in China, tobacco is an intolerance continuous cropping crop. The continuous cropping will reduce the yield and quality of tobacco. But due to limitations in regional and economic conditions, the continuous cropping of tobacco has become a normal pattern in many areas. ${ }^{[1]}$ Soil is a kind of organism. The change of soil microenvironment directly influences the mineralization of soil organic matters and the transformation of nutrient forms. ${ }^{[2]}$ Soil microorganism plays an important role in maintaining the quality of soil. Soil microorganism is an essential biological index in soil quality evaluation. As a new source of pigment, marigold has high economic values; ${ }^{[3]}$ meanwhile, it can control and kill microorganisms, ${ }^{[4-5]}$ and effectively control root knot nematodes. ${ }^{[6-8]}$ In this study, Yunyan 87 was the research material. The second generation high throughput sequencing technology was used to study the effect of marigold planting on microbial diversity of tobacco planting soil under cultivation patterns of marigold and tobacco inter planting, and marigold single planting. The aim of this paper was to 
provide technical reference for the adjustment of tobacco planting patterns and the green planting of tobacco.

\section{Research Materials and Methods}

\subsection{Sample collection.}

The tested tobacco variety was Yunyan 87. The three treatment methods were single cropping tobacco, tobacco intercropping with marigold and single cropping marigold. The field which had been used in tobacco planting for consecutive three years in Qujing, Yunnan province was selected as the experiment field. During the squaring stage, five-point sampling method was used to collect soil samples. Samples were stored in ziplock bags and numbered. Rhizosphere soil sample from the single cropping tobacco field was numbered as AB3.2; rhizosphere soil sample from the tobacco intercropping with marigold field was numbered as BB3.2; rhizosphere soil sample from the single marigold planting field was numbered as CC3.2.

\subsection{Extraction of total DNA.}

According to the instruction book of genomic DNA extraction kit produced by E.Z.N.A. Soil DNA Kit, total DNA of soil microorganism was extracted. Then the purity and concentration of DNA were examined by agarose gel electrophoresis. ${ }^{[9]}$

\subsection{PCR amplification.}

The diluted genomic DNA was used as a template. PCR was carried out through specific primers from 16S-V4 and ITS1 regions and equipped with Barcode. ${ }^{[10]}$

\subsection{PCR purification and computer sequencing.}

PCR products were detected by $2 \%$ agarose gel electrophoresis; the target band products were recovered through gel recovery kit produced by Qiagen Company. Sequencing was commissioned to Beijing Novogene Corporation.

\section{Research Results and Analysis}

\subsection{Sample diversity index analysis.}

After sequencing the 16S rRNA gene in V4 region of bacterial, 43319, 33810 and 51550 effective sequences were found in the three samples of AB3.2, BB3.2 and CC3.2 respectively. Under the 97\% similarity level, these effective sequences could be divided into 2095, 2217 and 2085 OTUs respectively. The Chao1 index order was CC3.2 > AB3.2 > BB3.2; the Shannon index order was BB3.2 > AB3.2 > CC3.2.

After the sequencing fungi in ITS1 region, 20159, 31305 and 26376 effective sequences were found in the 3 samples of AB3.2, BB3.2 and CC3.2 respectively. Under the 97\% similarity level, these effective sequences could be divided into 342, 320 and 291 OTUs respectively. The order of Chao1 index was BB3.2 > AB3.2 > CC3.2; Shannon index order was BB3.2 > AB3.2 > CC3.2.

\subsection{The distribution characteristics of microbial community.}

Bacteria found in three samples were distributed in 11 phyla, namely Proteobacteria, Acidobacteria, Actinobacteria, Gemmatimonadetes, Chloroflexi, Planctomycetes, Bacteroidetes, Thaumarchaeota, Nitrospirae, Verrucomicrobia and Firmicutes. Proteobacteria, Acidobacteria and Actinobacteria were dominant bacteria in the three samples. They accounted for $41.41 \%, 19.09 \%$ and $11.53 \%$ of total bacteria in sample AB3.2; accounted for $43.89 \%, 14.72 \%$ and $16.65 \%$ of total bacteria in sample BB3.2; accounted for $35.83 \%, 16.29 \%$ and $21.17 \%$ of total bacteria in sample CC3.2. From the perspective of genus, dominant bacteria of AB3.2 were distributed in Kaistorbacte (3.64\%), Marinobacter (3.47\%), Nitrososphaera (2.44\%), Alcanivorax (1.75\%), Rhodoplanes (1.22\%), Lysobacter (0.59\%), Thermomonas (0.61\%), Streptomyces (0.38\%), Pseudonocardia 
(0.37\%) and Hydrogenophaga (0.33\%). Dominant bacteria of BB3.2 were distributed in Kaistorbacte (3.95\%), Marinobacter (3.29\%), Nitrososphaera (2.33\%), Rhodoplanes (1.28\%), Hydrogenophaga (0.77\%), Thermomonas (0.75\%), Lysobacter (0.74\%), Streptomyces (0.69\%), Pseudonocardia (0.63\%) and Alcanivorax (0.34\%). Dominant bacteria of CC3.2 were distributed Kaistorbacte (3.92\%), Marinobacter (3.29\%), Nitrososphaera (1.53\%), Rhodoplanes (1.34\%), Streptomyces (1.13\%), Pseudonocardia (0.75\%), Thermomonas (0.71\%), Lysobacter (0.66\%), Alcanivorax (0.44\%) and Hydrogenophaga (0.13\%). At the level of genus, the dominant bacteria of three samples were similar; three kinds of bacteria with higher abundances were Kaistorbacte, Marinobacter and Nitrososphaera. There were only small differences in the abundances of the same genus. The content of Nitrososphaera in CC3.2 was lower than that in AB3.2 and BB3.2 by $0.80 \%$ to $0.91 \%$.

Fungi found in three samples were distributed in 4 phyla, namely Ascomycota, Basidiomycota, Zygomycota and Chytridiomycota. Ascomycota were the dominant flora of all the 3 samples of AB3.2, BB3.2 and CC3.2, with the abundances of $86.37 \%, 86.13 \%$ and $89.52 \%$. Ascomycota was followed by Basidiomycota, with the abundances of $9.53 \%, 7.01 \%$ and $6.30 \%$. Few Chytridiomycota were found in BB3.2; the abundance was only $0.21 \%$. In AB3.2 and CC3.2 no Chytridiomycota could be found. From the perspective of genus, dominant fungi of AB3.2 were distributed in Tremellomycetes sp (9.37\%), Chaetomiaceae sp (6.25\%), Corynascella (6.24\%), Retroconis (4.41\%), Pulvinula (4.12\%), Penicillium (4.00\%), Chaetomium (3.89\%), Sordariales sp (3.75\%), Ascomycota sp (2.26\%) and Aspergillus (0.89\%). Dominant bacteria of BB3.2 were distributed in Chaetomiaceae sp (8.22\%), Aspergillus (7.70\%), Tremellomycetes sp (4.75\%), Chaetomium (4.47\%), Retroconis (4.22\%), Sordariales sp (4.09\%), Penicillium (2.52\%), Ascomycota sp (2.38\%), Pulvinula (0.13\%) and Corynascella (0.04\%). Dominant bacteria of CC3.2 were distributed in Chaetomiaceae sp (8.67\%), Corynascella (7.39\%), Tremellomycetes sp (6.08\%), Aspergillus (5.61\%), Retroconis (5.26\%), Sordariales sp (5.03\%), Chaetomium (4.92\%), Penicillium (4.32\%), Ascomycota sp (3.15\%) and Pulvinula (0.08\%). At the level of genus, the three samples had similar dominant fungi, but the abundances of fungi varied greatly. After the planting of marigold, the abundance of Aspergillus increased from the original number of $0.89 \%$ to values between $5.61 \%$ and $7.70 \%$. Compared with single cropping of tobacco, marigold inter planting with tobacco increased the abundance by 8.6 times. The abundance of Sordariales sp rose from the original number of $3.75 \%$ to values between $4.09 \%$ and $5.03 \%$ after marigold cultivation. The abundance of Corynascella was between $6.24 \%$ and $7.39 \%$ under monoculture, and sharply decreased to $0.04 \%$ under the condition of tobacco inter planting with marigold. The decrease rate was over $99 \%$.

\section{Conclusion and Discussion}

In this experiment, the second generation high-throughput sequencing technology was used to study the effects of two cultivation patterns, single cropping marigold and tobacco intercropping with marigold, on the soil microbial diversity compared with single cropping tobacco. The results showed that the sequence of bacterial abundance was CC3.2 > AB3.2 > BB3.2; the sequence of bacterial diversity was BB3.2 > AB3.2 > CC3.2. The sequence of fungal abundance was BB3.2 > AB3.2 > CC3.2; the sequence of fungi diversity was BB3.2 > AB3.2 > CC3.2. It could be seen that the pattern of tobacco intercropping with marigold reduced the bacterial abundance of tobacco planting soil, while increased the fungal abundance and microgram diversity. The monoculture of marigold increased the bacterial abundance, while reduced fungal abundance and microgram diversity.

Proteobacteria, Acidobacteria and Actinobacteria were dominant bacteria in the three samples. They accounted for $41.41 \%, 19.09 \%$ and $11.53 \%$ of total bacteria in sample AB3.2; accounted for 43.89\%, $14.72 \%$ and $16.65 \%$ of total bacteria in sample BB3.2; accounted for $35.83 \%$, $16.29 \%$ and $21.17 \%$ of total bacteria in sample CC3.2. At the level of genus, the dominant bacteria of three samples were similar; three kinds of bacteria with higher abundances were Kaistorbacte, Marinobacter and Nitrososphaera. There were only small differences in the abundances of the same 
genus. The content of Nitrososphaera in CC3.2 was lower than that in AB3.2 and BB3.2 by $0.80 \%$ to $0.91 \%$.

Ascomycota was the dominant fungi in all the 3 samples of AB3.2, BB3.2 and CC3.2 with abundances of $86.37 \%, 86.13 \%$ and $89.52 \%$. Ascomycota was followed by Basidiomycota with the abundances of $9.53 \%, 7.01 \%$ and $6.30 \%$. Few Chytridiomycota were found in BB3.2; the abundance was only $0.21 \%$. In AB3.2 and CC3.2 no Chytridiomycota could be found. At the level of genus, the three samples had similar dominant fungi, but the abundances of fungi varied greatly. After the planting of marigold, the abundance of Aspergillus increased from the original number of $0.89 \%$ to values between $5.61 \%$ and $7.70 \%$. Compared with single cropping tobacco, marigold inter planting with tobacco increased the abundance by 8.6 times. The abundance of Sordariales sp rose from the original number of 3.75\% to values between $4.09 \%$ and $5.03 \%$ after marigold cultivation. The abundance of Corynascella was between $6.24 \%$ and $7.39 \%$ under monoculture, and sharply decreased to $0.04 \%$ under the condition of tobacco inter planting with marigold. The decrease rate was over $99 \%$.

The comprehensive analysis shows that, the pattern of tobacco intercropping with marigold can improve the microbial diversity of tobacco planting soil. In further research, it is necessary to find out whether the inter-cropping system can improve the quality of tobacco leaves and comprehensive benefits, so as to provide technical reference for solving problems in continuous tobacco cropping.

\section{Acknowledgements}

This paper is supported by National Natural Science Foundation of China (41361056, 31460491, 31660426); Joint Special Fundamental Research of Local Universities in Yunnan Province (2017FH001-041, 2017FH001-035, 2017FH001-005); Talent Introduction Program of Kunming University (YJL14005); Key Disciplines (Ecology) Project of Yunnan Education Department (05000511311); Open Fund Project of Key Laboratory of Characteristic Biological Resources Development and Utilization in Yunnan Province (GXKZ201716); China National Tobacco Corp Science and Technology Project (2018530000241020, 2018530000241016).

\section{References}

[1] X.F. Li, Y.M. Wang, C.L. Xu, et al., Nematicidal activity of Marigold on tobacco, J. Journal of Anhui Agricultural Science. 45 (2017).

[2] S.X. Zhang, W.M. Guo, H.X. Li, et al., Advance in continuous cropping problems of tobacco, J. Soils. 47 (2015).

[3] H.Y. Wei, L.R. Guan, Y. Wang, et al. Effect of marigold diversified cropping with Angelica on fungal community in soils, J. Plant Protection. 41 (2015).

[4] T.Y. Zhang, Y.S. Li, Y.N. Guo, et al., Effect of marigold intercropping density on the control of Root-knot nematodes, growth and yield of cucumber, J. Chinese Journal of Biological Control. 30 (2014).

[5] T.Y. Zhang, Effect of Lime Nitrogen and Marigold on Cucumber Root Knot Nematode Control under Organic Substrate Cultivation, Chinese Academy of Agricultural Sciences, 2014.

[6] D.M. Chen, Ecological Regulation Mechanism of Diversified Cultivation on Obstacles in Continuous Cropping Tobacco, Fujian Agriculture and Forestry University, 2010.

[7] J.B. Liu, X. Song, J.S. Wang, Studies on bioactive Flavone from Tagetes root against Watermelon Fusarium wilt, J. Journal of Shanxi Agricultural University (Nature Science Edition). 1 (2008).

[8] W.B. Wang, C.R. Guo, Action of the Different Extracts of Tagetes Patula on Fusarium osysporum Schl. f. sp vasinfectum (Atk.) Snyd. \& Hans, J. Journal of Shanxi Agricultural University (Nature Science Edition). 4 (2004). 
[9]Y.X. Liu, X. Li, K. Cai, et. al., Identification of benzoic acid and 3-phenylpropanoic acid in tobacco root exudates and their role in the growth of rhizosphere microorganisms, J. Applied Soil Ecology. 93 (2015).

[10] M. Cosme, S. Wurst, Interactions between arbuscular mycorrhizal fungi, rhizobacteria, soil phosphorus and plant cytokinin deficiency change the root morphology, yield and quality of tobacco, J. Soil Biology \& Biochemistry. 57 (2013). 